\title{
Margaret McCartney: Flibanserin for low sexual desire is not feminism
}

\author{
Margaret McCartney general practitioner, Glasgow
}

The UK Medicines and Healthcare Products Regulatory Agency will neither confirm nor deny it, but flibanserin is likely to be coming to British shores. Its maker, Sprout Pharmaceuticals, told me that it is "committed to working closely with other regulatory bodies outside the US . . to bring to market a safe and effective treatment for the millions of women around the world affected by distressing low sexual desire." The drug was licensed at the third attempt in the United States for the treatment of hypoactive sexual desire in premenopausal women. ${ }^{1}$

Ironically, a pressure group used the feminism argument to push for the US Food and Drug Administration's approval on grounds of equality (men have their drugs; we want ours), when feminism is in fact a reason to object to flibanserin. How can it be feminist for doctors to tell women what's normal and prescribe pills to control their sexual desire?

We are told that $43 \%$ of women have low libido. ${ }^{2}$ But women in this study were not asked whether their lack of interest was a problem for them-let alone an illness. Is experiencing no pleasure during sex a medical problem to be solved? What about a woman's relationship or the culture that surrounds her?

The intersection of psychiatry, feminism, and pharma has an uncomfortable history. The Diagnostic and Statistical Manual of Mental Disorders in 1952 described the "frigidity" of women, ${ }^{3}$ which was expanded and differentiated over time. By 2013 the manual described "female sexual interest/arousal disorder."

Some researchers have called on doctors to take responsibility for maintaining "sexual health," decrying "underdiagnosis and undertreatment" and noting pharmaceutical solutions (as well as declaring interests of consultancy to Sprout). ${ }^{5}$

But does flibanserin work? The FDA turned it down because one of two primary endpoints studied in two phase III studies, a daily diary of sexual desire, did not reach statistical significance.

A third trial asked women retrospectively about their sexual desire in the previous four weeks. Across the trials, 0.5 to 1 more "satisfactory sexual events" a month occurred with flibanserin than with placebo. Even if real life use reflects this, is it clinically significant? And is it worth the side effects: low blood pressure, fatigue, and somnolence?
Interaction with alcohol was assessed for side effects among only 23 men and two women. ${ }^{67}$ Flibanserin is taken daily, and alcohol is contraindicated because of concerns about hypotension. This suggests that women can never drink while taking it.

The message on Sprout's voicemail says that it needs no further investors at this time. Save your money: the drug may be only slightly better than placebo. This is a medical "solution" offered for the huge complexity of female sexuality. It short-changes women. Flibanserin is not feminism.

Competing interests: I have read and understood the BMJ policy on declaration of interests and declare the following interests: I'm an NHS GP partner, with income partly dependent on Quality and Outcomes Framework points. l've written two books and earn from broadcast and written freelance journalism. I'm an unpaid patron of Healthwatch. I make a monthly donation to Keep Our NHS Public. I'm a member of Medact. I'm occasionally paid for time, travel, and accommodation to give talks or have locum fees paid to allow me to give talks but never for any drug or public relations company. I was elected to the national council of the Royal College of General Practitioners in 2013 and am chair of its standing group on overdiagnosis. I have invested a small amount of money in a social enterprise, Who Made Your Pants?

Provenance and peer review: Commissioned; not externally peer reviewed.

Follow Margaret on Twitter, @mgtmccartney

Roehr B. FDA committee recommends approval for "female Viagra." BMJ2015;350:h3097. 2 Laumann EO, Paik A, Rosen RC. Sexual dysfunction in the United States: prevalence and predictors. JAMA 1999;281:537-44.

3 Angel K. The history of "female sexual dysfunction" as a mental disorder in the 20th century. Curr Opin Psychiatry 2010;23:536-41.

4 IsHak WW, Tobia G. DSM-5 changes in diagnostic criteria of sexual dysfunctions. Reprod Syst Sex Disord 2013;2:122, doi:10.4172/2161-038X.1000122.

5 Kingsberg SA, Rezaee RL. Hypoactive sexual desire in women. Menopause 2013;20:1284-300

6 Basson R, Driscoll M, Correia S. Flibanserin for low sexual desire in women: a molecule from bench to bed? EBioMedicine 2015;2:772-3.

7 Food and Drug Administration. FDA access data: flibanserin. www.accessdata.fda.gov/ drugsatfda_docs/label/2015/022526lbl.pdf. 
